\title{
A novel fractional order power factor measurement
}

\author{
Shahriar Rahman Fahim ${ }^{1} \cdot$ Sakib Shahrear Avro ${ }^{2}$ Subrata K. Sarker ${ }^{3} \cdot$ Sajal K. Das $^{2}$
}

Received: 2 July 2019 / Accepted: 7 November 2019 / Published online: 14 November 2019

(c) Springer Nature Switzerland AG 2019

\begin{abstract}
This paper presents the design and experimental implementation of a smart power factor metering system to measure the fractional order power factor of a load. The variation of grid frequency or power factor (PF) increases the utility charges. The proposed design provides an accurate PF measurement by using a real-time AC power triangle. The system is designed by proposing a novel embedded system based controller that enables reading the main-line frequency, load voltage, current and their phase differences. A converter is designed first to transform the high voltage and current to the controller. The converter consists of a potential transformer, a current transformer, high differential strobe terminal based comparator and a logic gate that detects the phase delay between the load voltages and currents. A set of parallel capacitors are interconnected with the load terminal for improved PF measurement. The improved power factor confirms the saving of the utility charges for inductive loads. The simulation and experimental results are demonstrated to validate the effectiveness of proposed metering system.
\end{abstract}

Keywords Power triangle $\cdot$ MATLAB $\cdot$ High differential comparator $\cdot X$-OR gate $\cdot$ Power factor $\cdot$ Proteus and Arduino

\section{Introduction}

Accurate power factor measurement from electrical energy is emerging topic to researcher because it distributes the electrical energy at reasonable cost. The cost of electrical energy increases due to power losses in transformer, transmission line and loads. Most of the loads are inductive where the current waveforms lagging the voltage. It consumes the reactive power which causes the real power to be less than the apparent power. The lower value of real power produces the poor power factor that can be used to measure the efficiency affecting by the phase lag [1-3]. A load with less power factor draws more current from the power line which is responsible for more losses.

The power factor directly relates to the system efficiency and performance. A large amount of load current results in lower voltage regulation of the system and provides a large joule loss in the power lines $[4,5]$. The lower voltage level generates heat in the motor windings that reduces the lifetime, reliability and performance of the machine $[6,7]$. The reduced system performance and efficiency generates poor value of power factor that results in increasing utility charge. The economic returns of the power factor can be easily noticeable in the lower utility charges. Another visible benefit of power factor is the released system capacity, decreasing line losses and improved voltage regulation. Though having lots of benefits of power factor, the power factor measuring device is not available with the range of consumers. With this in mind, the system introduces in this paper measures the power factor and plots a power diagram for further analysis. The proposed system also calculates the necessary capacitance needed to improve the power factor and improve it.

$\triangle$ Subrata K. Sarker, skshuvo138008@gmail.com; Shahriar Rahman Fahim, fahim.fact@gmail.com; Sakib Shahrear Avro, sakibavro.ruet@gmail.com; Sajal K. Das, das.k.sajal@gmail.com | 'Department of Electrical \& Electronic Engineering, Rajshahi University of Engineering \& Technology, Rajshahi 6204, Bangladesh. ${ }^{2}$ Department of Mechatronics Engineering, Rajshahi University of Engineering \& Technology, Rajshahi 6204, Bangladesh. ${ }^{3}$ Department of Electrical \& Electronic Engineering, Varendra University, Rajshahi, Bangladesh. 


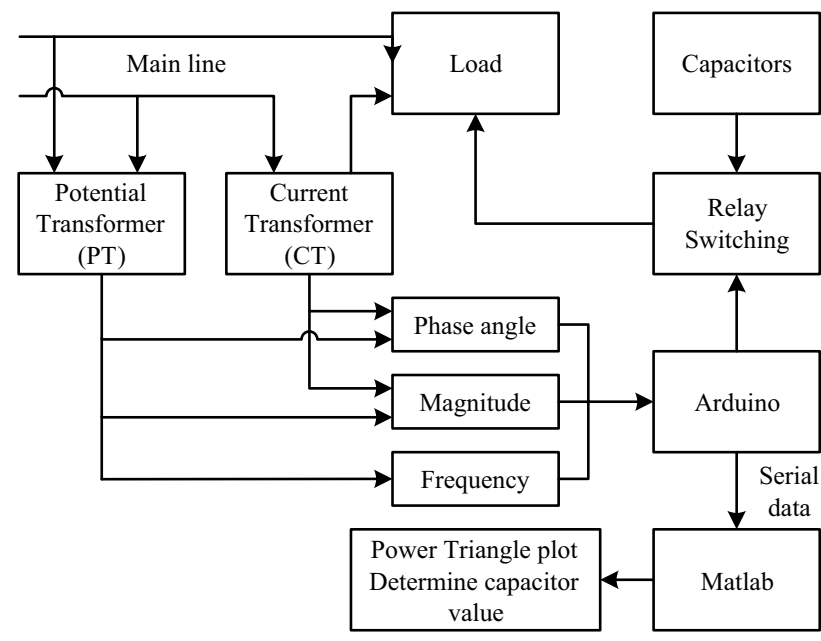

Fig. 1 Basic block diagram of the proposed system

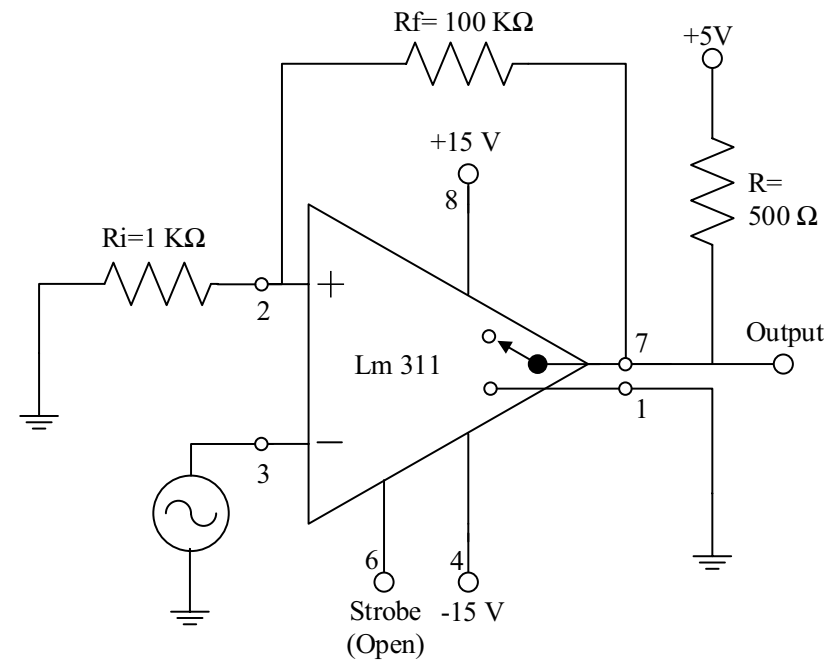

Fig. 2 A comparator circuit using LM 311

Figure 1 shows the basic block diagram of the proposed system. The system first converts the high value of load voltage and current to the proportional lower value. The conversion is done by using a PT of 20:1 trans ratio and a CT of 1:24 turns ratio. The output of CT and PT is fed into a comparator circuit designed with LM 311 IC, as shown in Fig. 2, for detecting the zero crossing. Its strobe terminal operation and low voltage handling capacity makes it possible to respond to the lower value of $C T$ output. As a result, no additional amplifier circuit is needed between $\mathrm{CT}$ and comparator circuit. An X-OR gate is used in the proposed system for detecting the lagging time pulse and phase delay between the voltage and current. A precision full wave rectifier is used to convert $A C$ to $D C$ voltage and current. The output from rectifier is passed through the Arduino and measures the equivalent voltage and current.
Arduino sends the data to the MATLAB through serial communication, and then the Matlab calculates active, reactive, and apparent power and plots a power triangle. The MATLAB also calculates the required capacitance value to improve the poor power factor to unity. In the case of improving the power factor, the system first estimates the necessary capacitance required to improve the power factor near unity. Three capacitors of values $5 \mathrm{uF}, 10 \mathrm{uF}$ and 20 $\mathrm{uF}$ are placed such a way that they can combine to form a new capacitance. A relay module performs the job of switching and combining the capacitors. After calculating the necessary capacitor value, the system searches for the minimum available capacitor and switch it.

The rest of this paper is organized as follows. Related works are discussed in Sect. 2 . Section 3 explains the problem formulation of power factor. In Sect. 4 the procedure of measuring power factor is described. The method of improving the power factor is expressed in Sect. 5. The experimental set up for proposed system is illustrated in Sect. 6. The experimental evaluation and cost analysis using the evaluated result is given in Sects. 7 and 8. This paper is concluded in Sect. 9.

\section{Related work}

Number of researches have been done to measure the power factor. In [8-10], a system is demonstrated which is used for measuring and correcting the power factor (PF). The system uses the required capacitance which is switched in or out by solid state relay and continuously monitors the PF. This system limits the application as a portable power factor meter. A PF measuring system based on Arduino is shown in [11] where the active power is calculated by taking the instantaneous value of voltage and current. It requires the high sampling rate. The higher sampling rate may cause sampling error, which affects the accuracy of measurement.

An ARM microcontroller based approach for monitoring the power factor is introduced in $[12,13]$. The drawback of this approach is its high implementation cost. In [14] power factor control unit based on Arduino is presented where a fixed line frequency is assumed. Moreover, the power line frequency may vary within a small range. The variation of frequency may affect the calculation of the power factor.

Motivated by above difficulties, this paper presents a smart power factor metering system for measuring and improving the PF of load. The measuring of PF is done by using a controller which controls the variation of frequency of main line, while the number of capacitors on load terminal with relays decrease the utility charge by improving the measuring PF. 
The contributions of this paper are as follows:

(i) Design of a novel fractional order PF measurement unit that measures the accurate fractional frequency from the main line.

(ii) Design of a high-speed differential strobe terminal base comparator that limits the use of the preamplifier circuit.

(iii) Number of capacitors are used on the load terminal to improve the measured power factor which limits the use of variable capacitor that may increase the development cost and makes the bulky system.

The proposed system is able to eliminate the error due to rounding the frequency like $50 \mathrm{~Hz}$. The aim of this work is to ensure the accurate PF by ploting a power triangle in the MATLAB environment. The power triangle is used to find the accurate PF by comparing the amount of power used in the load and consumed power from the transmission line.

\section{Problem formulation}

The power factor of the circuit $\cos (\phi)$ is measured by taking the phase difference $\phi$ between the voltage and current in an AC circuit. When the circuit is inductive, the current lags behind the voltage and the power factor is mentioned to as lagging. Most of the residential loads are inductive in nature and therefore have low lagging power factor. The power factor can also be calculated in terms of power drawn by the AC circuit. The ratio of active power to the apparent power is referred to as power factor. The relation between the active, reactive and apparent power is shown in Fig. 3 and discussed in (1), (2) \& (3) as follows:

$P=V \times I \times \cos (\phi)$

$Q=\sqrt{S^{2}-P^{2}}$

$S=V \times I$

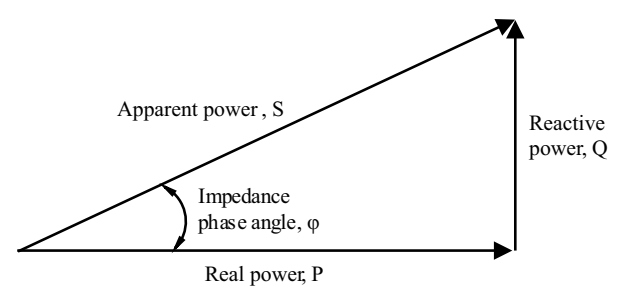

Fig. 3 Relation between the active, reactive and apparent power
For measuring the phase angle, the sinusoidal output from CT and PT are fed into two different comparator circuit. LM 311 is chosen to make a comparator circuit as shown in Fig. 2 because of its strobe terminal operation and faster response time of $165 \mathrm{nS}$ [15]. The result from strobe terminal acts like a switching output and connecting between a voltage source $V_{+}$and ground. When the strobe switch is open, the LM 311 operates normally and gives the positive output voltage $V_{+}$for negative values of the input signal and $0 \mathrm{~V}$ for the positive values as reported in Fig. 4.

Figure 5 presents the phase angle generation using the time delay between the voltage and the current. A simulation result with the input and output wave shape of comparator circuit is presented in Fig. 6 . Here the sinusoidal output of channel A shows the input wave shape and channel $B$ shows the output of the comparator circuit. From Fig. 5, it is observed that the comparator generates an output of +5 volt for every negative value and returns 0 volts for the positive value of input wave. The switching outputs are passed through the X-OR gate to detect the time delay between the voltage and current. This logic gate detects the inequality between two pulses and generates another pulse. The on time of this pulse denotes the time delay between the voltage and current.

Power factor improvement refers to the technology that recuperates the power factor close to unity as it is economically viable. This is performed by the addition of capacitors in parallel with the load which recompenses for the reactive power of the inductive load and therefore decrease the freight on the supply. The capacitors in the power factor improvement system draw a current that leads over the voltage and generates the leading power factor. The leading capability of capacitor decreases the lagging power factor proportionately in the circuit that results in improve the overall power factor for the load. In Fig. 7, S1 shows the apparent power before power factor improvement and S2 shows that afterward where S1 leads the S2. Here, Qc is the reactive power supplied by the capacitor.

\section{Power factor measurement}

This section presents the brief discussion of the power factor measurement by using phase difference between the voltage and current of an inductive load. The phase difference is obtained by measuring the time delay with the help of Arduino board. After measuring the time delay, the phase difference between the voltage and the current is measured. The relation between the time delay and the phase difference is described by the following equations: 
Fig. 4 Strobe terminal base operation for comparator of Fig. 2

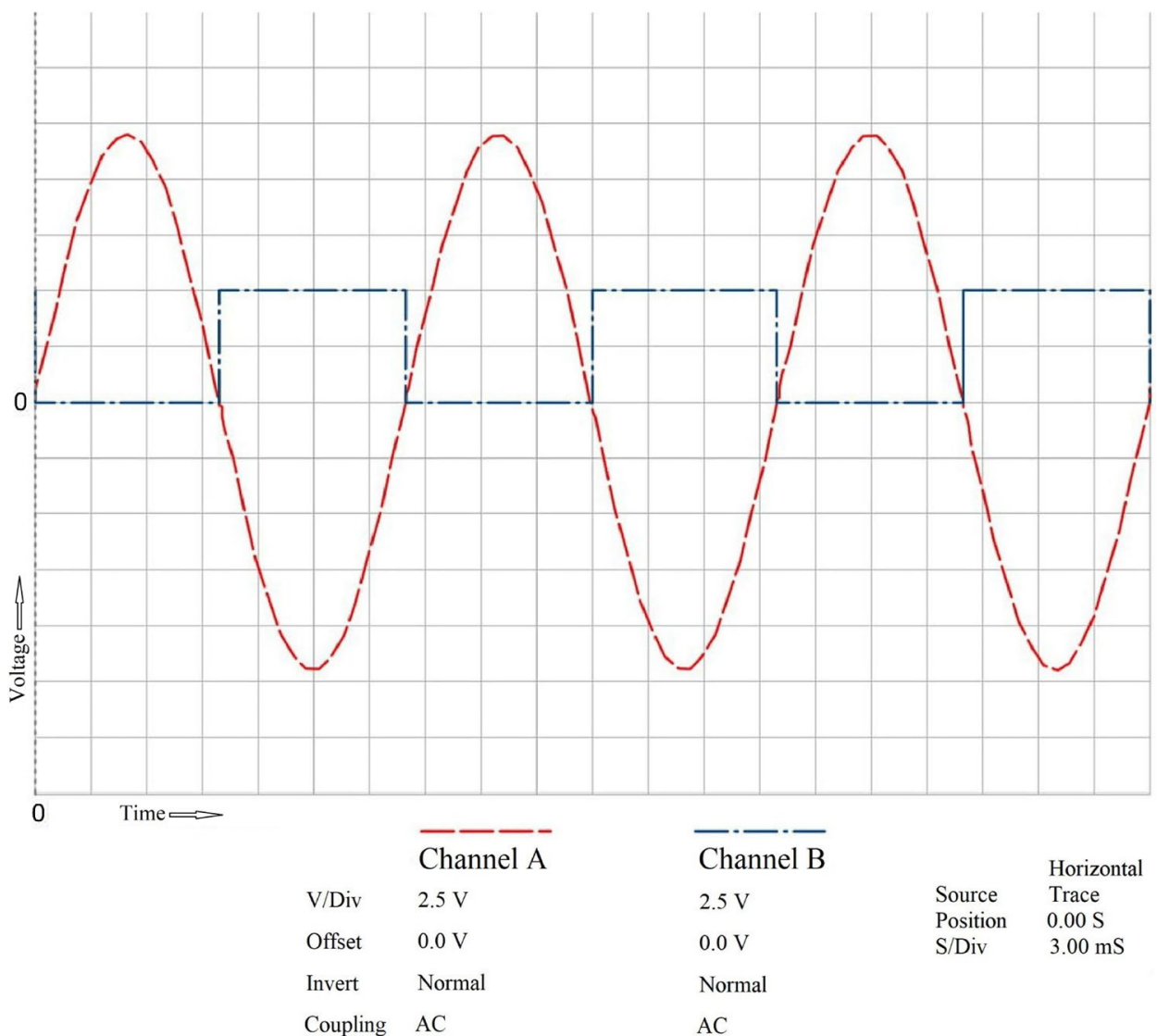

$\phi_{D}=2 \times 180 \times f \times T_{D} \times 10^{-6}$

$\phi_{R}=\frac{\pi}{180} \phi_{D}$

Here, $\phi_{D}$, Phase difference in degrees; $T_{D}$, Time delay in $\mu S$; $f$, Frequency in $\mathrm{Hz} ; \phi_{R}$, Phase difference in radians.

A flow chart of the programme for measuring the power factor is shown in Fig. 8. The time delay between the voltage and the current is measured using the pulseln() function of Arduino. The process of the pulseln() function is that it waits until the rising edge of X-OR output is detected. While detecting the signal from low to high, it stocks the time in its memory using the function micros(). After that, a recursive function is called which call itself again and again until detecting the falling edge. If the signal goes low, it deducts the current time from the starting time. This process returns the on-time duration of the pulse. Based on this on-time duration, the power factor is then evaluated using (4) and (5). After that, taking the cosine of the phase angle and returns the power factor. The obtained power factor is then put in (1) and calculates the required active power for the load. From (1), it is seen that the load voltage and current also needs to be measured in order to calculate the active power. The proposed system is able to measure the load voltage and current. The measurement process of load voltage and current is discussed in the next section.

\subsection{Voltage measurement}

For measuring the voltage across the load, the output from the potential transformer is given to a rectifier circuit. $A$ precision full wave rectifier circuit as shown in Fig. 9 is chosen for the rectification purpose.

The rectifier circuit excludes the additional voltage drop across the diode and rectifies low value of $A C$ signal by adding the large gain of OP-Amp. A voltage divider is employed to convert the high value of $D C$ signal into Arduino compatible form. The ratio of Arduino input to the total DC voltage is 0.4166 used for the proposed system. As the Arduino uses 10-bit ADC, it gives 0-1023 (1024 steps) for a $0-5 \mathrm{~V}$ input. After using this step, the Arduino calculates the load voltage by using the following equations:

$V_{D C}=\frac{A D C_{\text {reading }} \times 0.00488}{0.4166}$ 


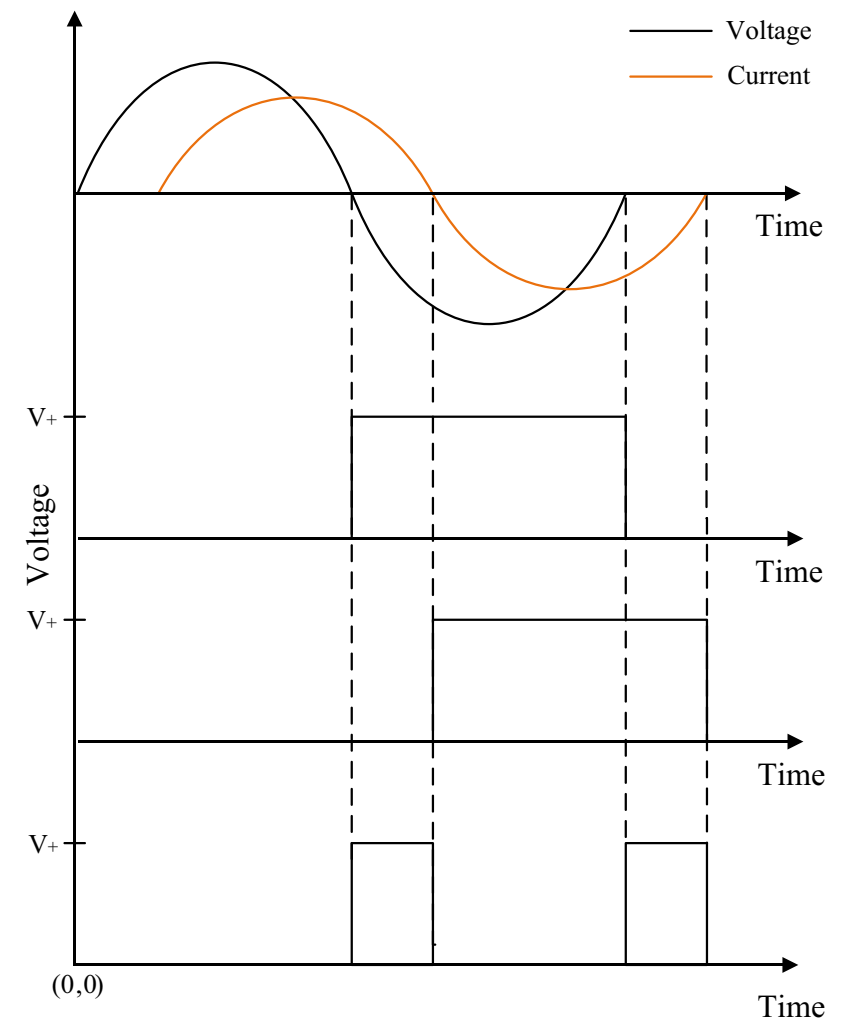

Fig. 5 Phase angle generation using the time delay between the voltage and the current

$V_{R M S}=\frac{\pi \times V_{D C}}{2 \sqrt{2}}$

$V_{\text {load }}=V_{R M S} \times N_{P T}$

Here, $V_{D C}$, Rectified DC voltage; $V_{R M S}$, RMS value of AC voltage at PT secondary; $V_{\text {load }}$, Load voltage; $N_{P T}$, Transformer ratio of $\mathrm{PT}$.

A flow chart of the programme for AC voltage measurement is reported in Fig. 10. For measuring the AC Voltage, Arduino waits for the voltage pin to go high. When the voltage pin goes high, the programme starts sampling for 1000 times. This is done by using a "for" loop. Inside the "for" loop, the programme samples the analog input from the rectifier circuit and stocks it into the memory. When the " for" loop is executed, the average value is computed by summing all the sampled data and dividing it by 1000 for obtaining the $A D C_{\text {reading. }}$. After completing this step, the load voltage is calculated by using (6)-(8).

\subsection{Current measurement}

A current transformer (CT) of 1:24 turns ratio is used to obtain the high value of load current. As the output of $\mathrm{CT}$ is a current signal, it converts the current signal into a voltage signal. The required conversion is done by adding a 100-ohm resistance at the secondary windings of the CT. The converted signal is applied to the full wave rectifier circuit. Arduino reads the voltage in the similar way used in voltage measurement and calculates load current by using the following equations:

$V_{D C}=\frac{A D C_{\text {reading }} \times 0.00488}{0.4166}$

$V_{R M S}=\frac{\pi \times V_{D C}}{2 \sqrt{2}}$

$I_{R M S}=\frac{V_{R M S}}{100}$

$I_{\text {load }}=I_{\text {RMS }} \times N_{C T}$

Here, $I_{R M S}$, RMS value of AC current at CT secondary; $I_{\text {load }}$ Load current; $N_{C T}$, Transformer ratio of CT.

\subsection{Frequency measurement}

The transmission line frequency is not exactly $50 \mathrm{~Hz}$ in an ideal case. It may vary between 49 to $51 \mathrm{~Hz}$. Thus, for better accuracy, the measurement of frequency is needed rather than assume it. The output of the comparator circuit is directly fed to the Arduino. Arduino then measures the on-time duration of the pulse output from the comparator circuit using pulseln() function. The time period of the original sinusoidal signal is twice the on-time duration of that pulse. This duration is then converted into frequency using the below equations:

$T=t_{\text {on }} \times 2$

$f=\frac{1}{T}$

Here, $t_{o n}$ On time duration of comparator pulse output; $T$, Time period; $f$, frequency.

A flow chart of the frequency measurement is shown in Fig. 11. The flow chart is similar as the power factor measurement except that the input of the programme is 
Fig. 6 Comparators output of Fig. 2

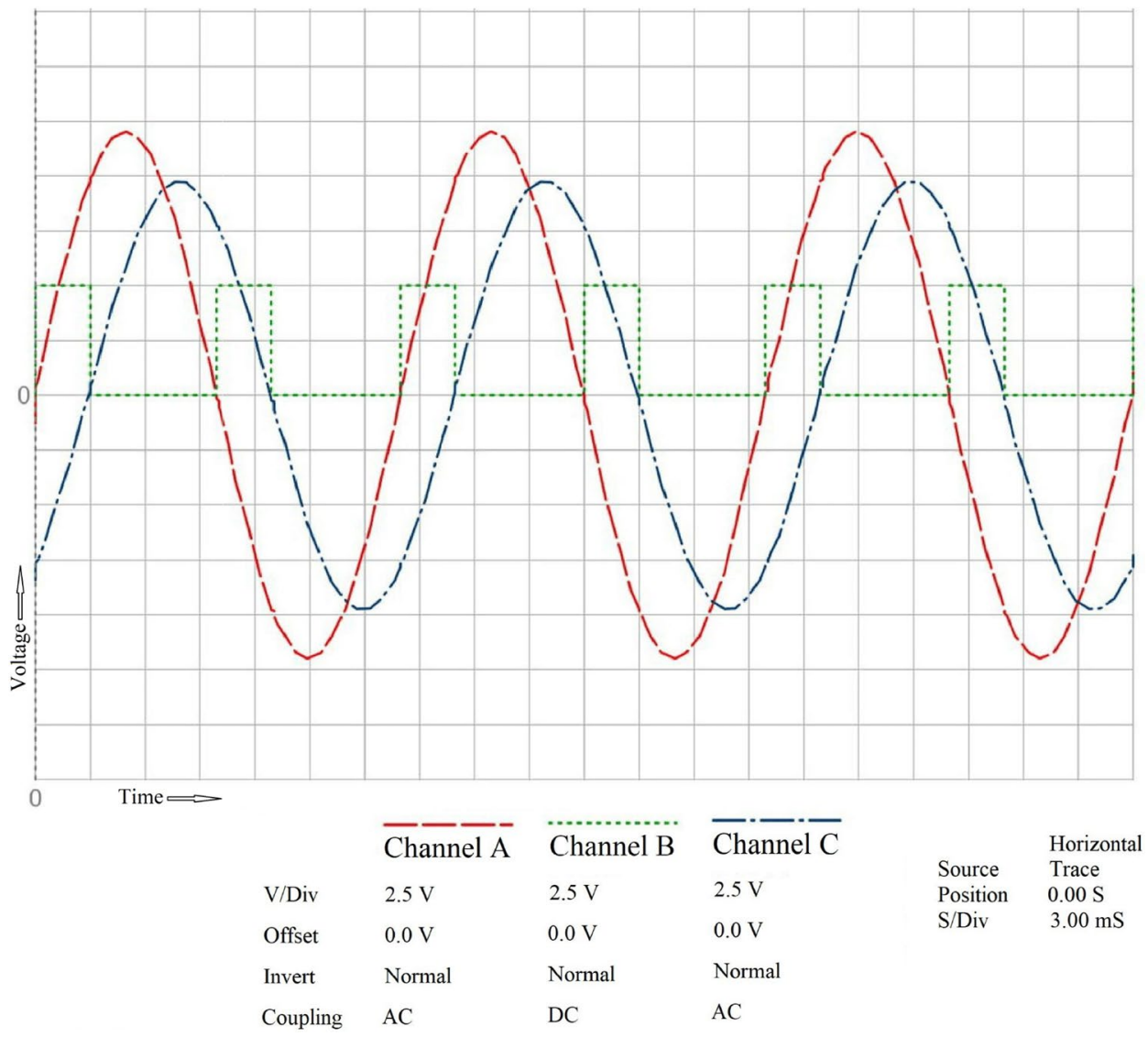

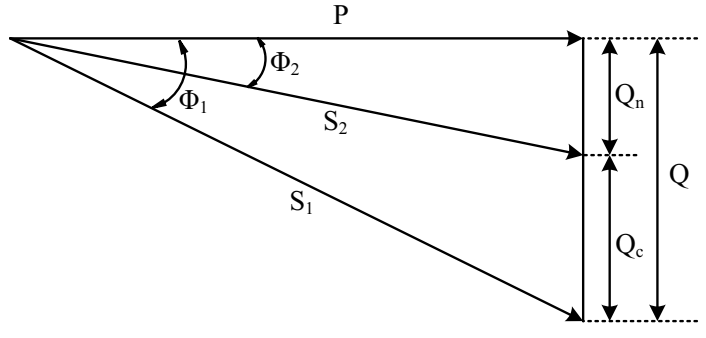

Fig. 7 Relation of lagging phase angle for power factor improvement

given from the output of the comparator. After measuring the on time of the comparator output as discussed in the power factor measurement section the frequency is then calculated from the Eqs. (13) and (14). The actual measured frequency of power line is presented in Fig. 12.

\section{Power factor improvement}

In this section, the procedure of power factor improvement is presented. At the beginning of the power factor improvement the system first decides if the power factor is less than 0.9. If the condition approves, the system then
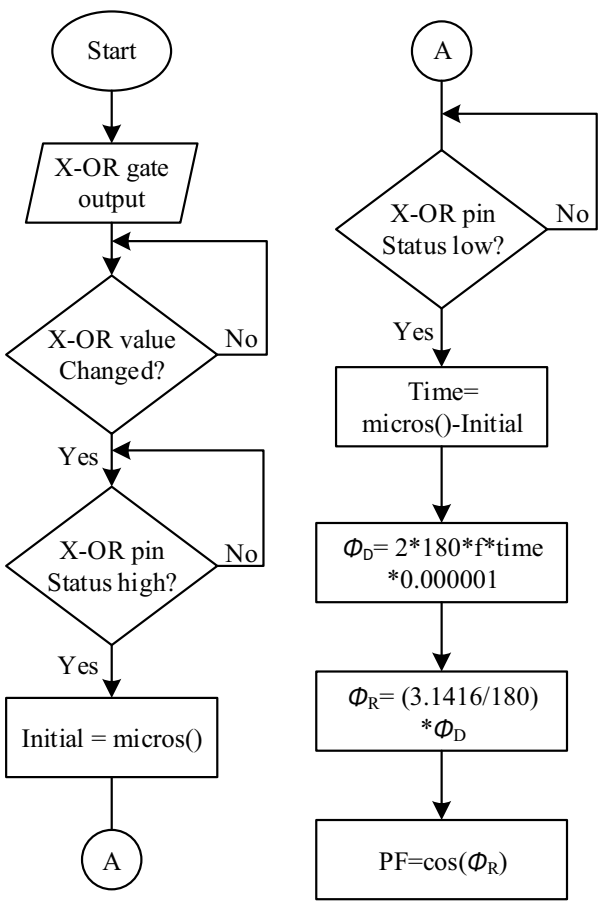

Fig. 8 Flow chart for power factor measurement 


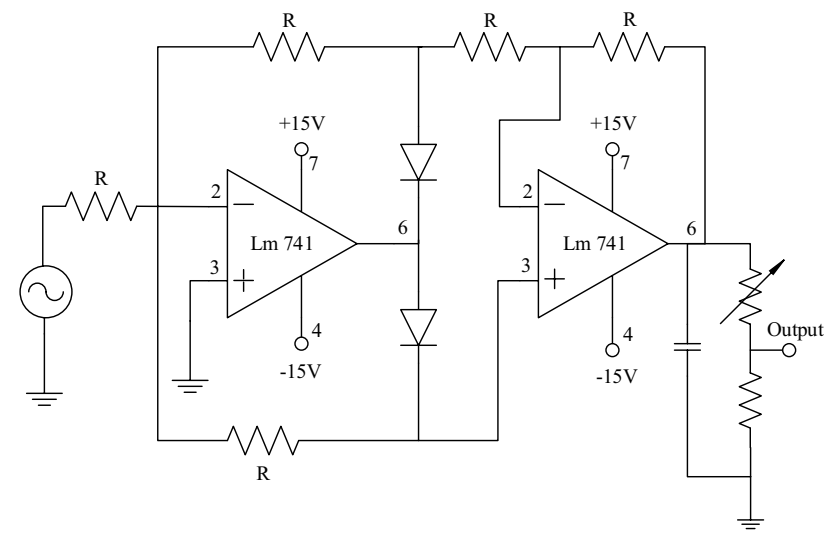

Fig. 9 A precision circuit diagram of full wave rectifier
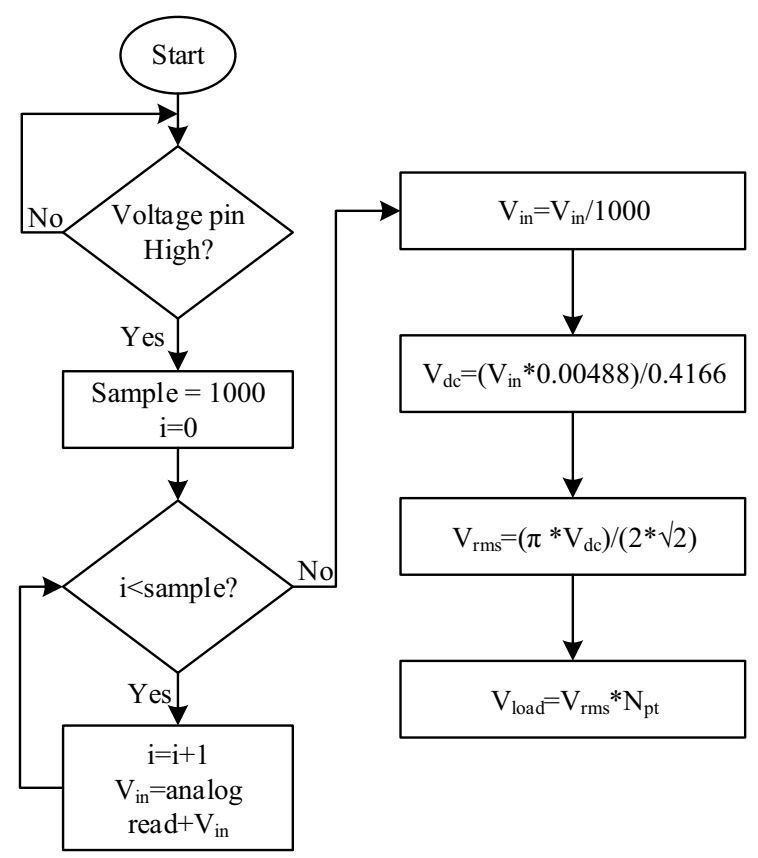

Fig. 10 Flow chart for load voltage measurement

calculates the KVar rating of the capacitor and then the $\mathrm{UF}$ rating using the Eqs. (1) and (2).

$T_{1}=\cos ^{-1}(P F)$

$T_{2}=\cos ^{-1}\left(P F_{D}\right)$

KVar $=P \times\left(\tan \left(T_{1}\right)-\tan \left(T_{2}\right)\right)$
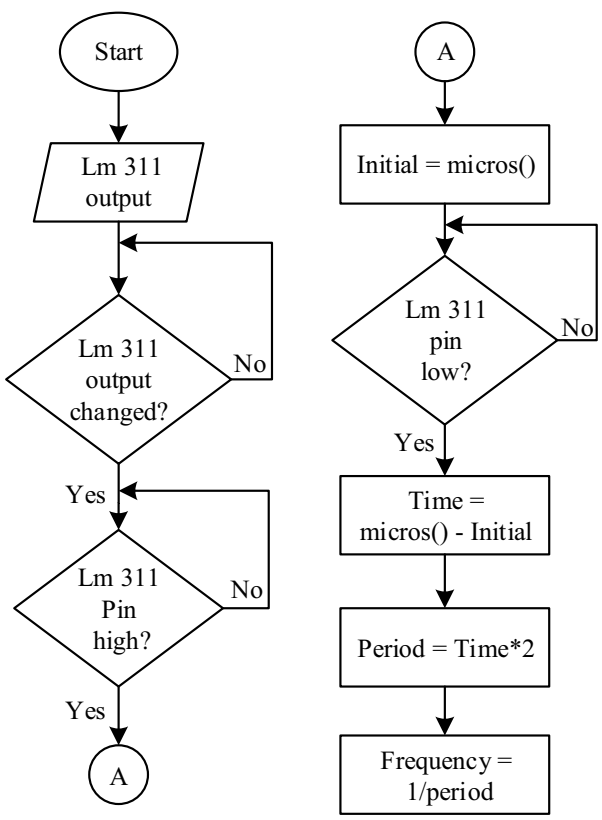

Fig. 11 Flow chart for frequency measurement

$$
\begin{aligned}
& T=\text { Time period } \\
& f=\text { frequency }
\end{aligned}
$$

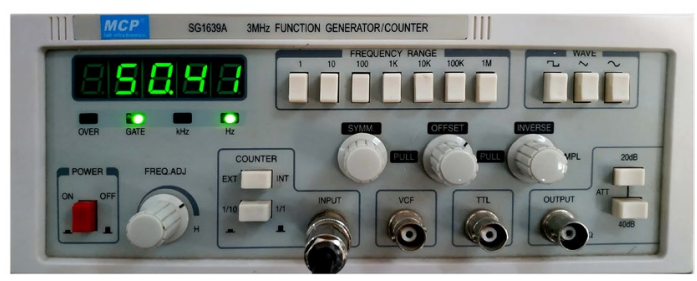

Fig. 12 Frequency measure using frequency counter

$$
C=\frac{K V a r}{2 \times \pi \times f \times V_{\text {load }}^{2}}
$$

Here, $P F$, Current power factor; $P F_{D}$, Desired power factor; $C$ Calculated capacitance.

Figure 13 presents the flow chart of the power factor improvement where the required capacitance is calculated for improving the power factor. After successfully calculating the capacitor value, the proposed system applies the minimum available capacitor across the load terminal. Three relays $\mathrm{R} 1, \mathrm{R} 2, \mathrm{R} 3$ are employed to switch the available capacitor across the load terminal which identify the minimum capacitance value for accurate power factor. 


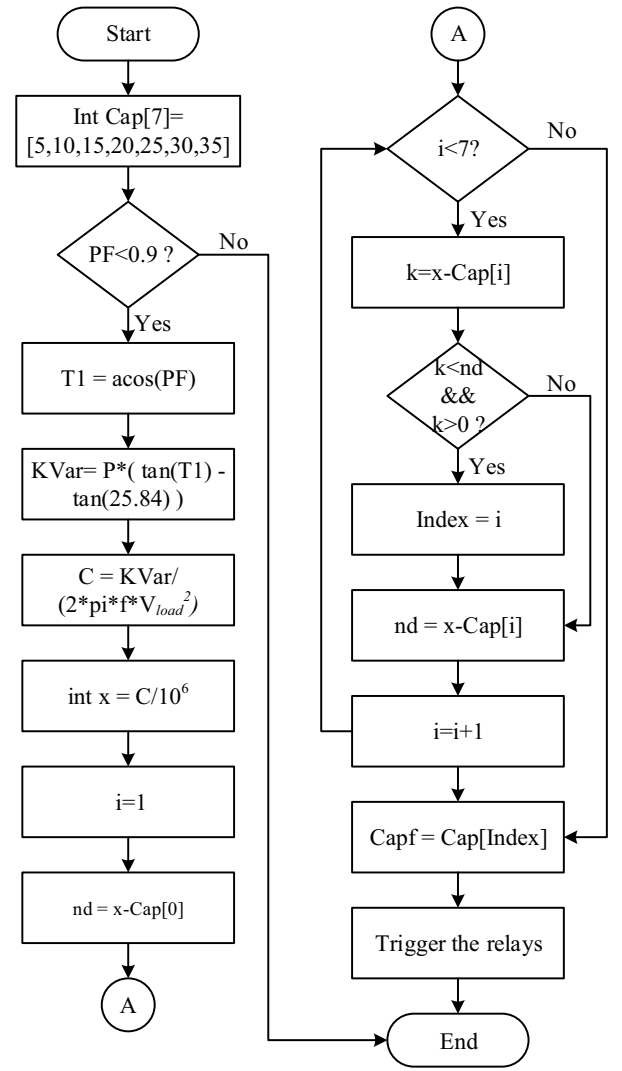

Fig. 13 Flow chart for the power factor improvement

Table 1 Field test of required capacitor value using GPS units with relay module

\begin{tabular}{llll}
\hline Capacitance & \multicolumn{3}{l}{ Relay } \\
\cline { 2 - 4 } & R1 & R2 & R3 \\
\hline 5 & 1 & 0 & 0 \\
10 & 0 & 1 & 0 \\
15 & 1 & 1 & 0 \\
20 & 0 & 0 & 1 \\
25 & 1 & 0 & 1 \\
30 & 0 & 1 & 1 \\
35 & 1 & 1 & 1
\end{tabular}

The relay logic and the available capacitor value is listed in Table 1. Here, the value 1 and 0 represents the "ON" and "OFF" state of relay respectively.

\section{Experimental setup}

This section presents the experimental setup for measuring the power factor as shown in Fig. 14. The setup consists of a power board, frequency counter, computer, oscilloscope, potential transformer and load. A $200 \mathrm{~W}$ hair drier

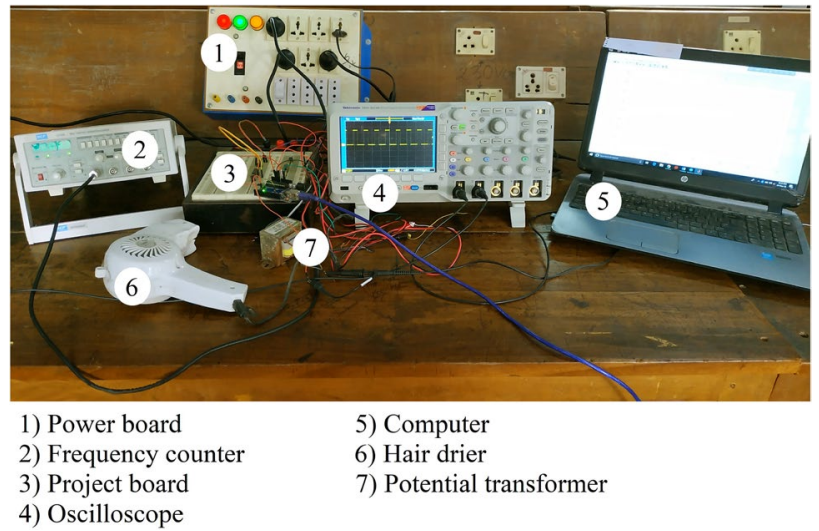

Fig. 14 Experimental set up for power factor measurement

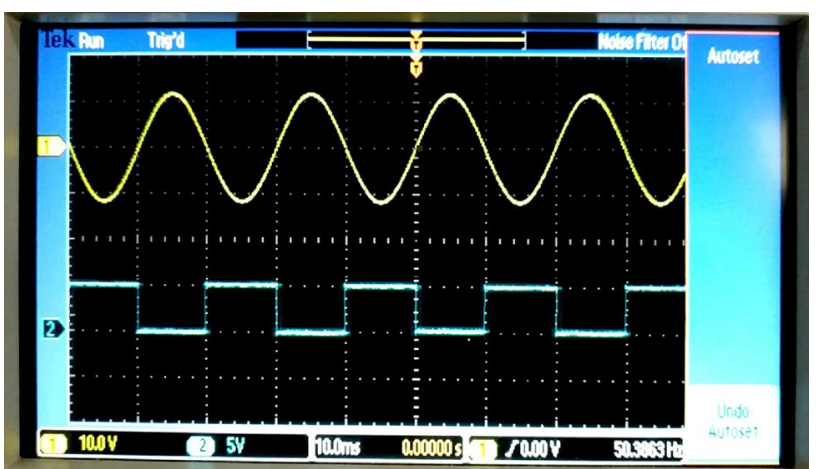

Fig. 15 Generation of pulse wave using high differential comparator

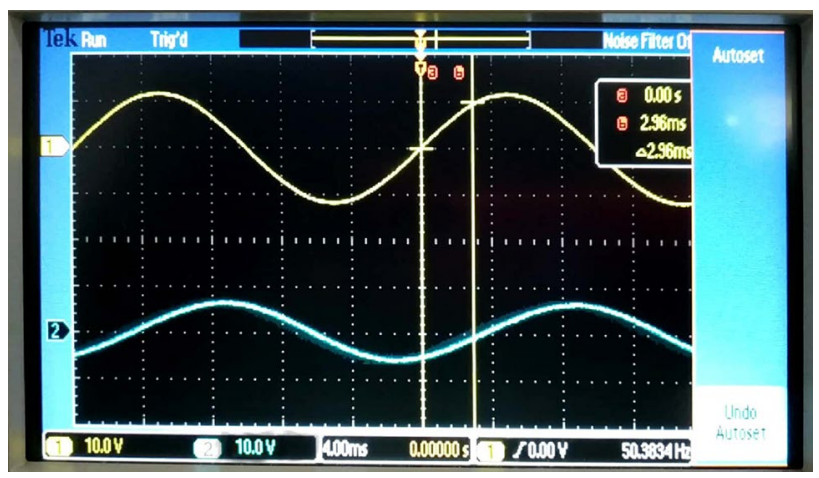

Fig. 16 Experimental output of the proposed system

is used as an inductive load which contains a motor that shows dynamic behavior along with the time. The function of frequency counter is to measure the actual frequency of main line. A computer is used for plotting the power triangle curve with the help of MATLAB that provides the support of analyzing of power factor. The wave shape of voltage, current, and time difference are observed in 
Table 2 Quantitative measurement using the conventional system

\begin{tabular}{ll}
\hline Parameter & Measured value \\
\hline Power factor & 0.598 \\
$V_{\text {load }}$ & $200 \mathrm{~V}$ \\
$I_{\text {load }}$ & $1.08 \mathrm{~A}$ \\
Active power & $129.17 \mathrm{~W}$ \\
Apparent power & $216 \mathrm{VA}$ \\
Reactive power & $173.12 \mathrm{Var}$
\end{tabular}

an oscilloscope. The performance of proposed system is evaluated by comparing its output and the measuring instrumental output.

\section{Performance analysis}

\subsection{AC power measured by the measuring device}

The power factor, frequency, and the AC power are first measured from the instrumental data. The performance of the proposed system with an inductive load is verified in Fig. 15 and Fig. 16. Here, the channel-1 (yellow wave) presents the voltage wave and channel-2 (blue wave) presents the current wave. From Fig. 16, it is seen that the time delay between voltage and current is $2.96 \mathrm{~ms}$. The frequency counter gives the accurate line frequency value of $50.41 \mathrm{~Hz}$ as shown in Fig. 12 and the phase difference between voltage and current is $53.716^{\circ}$ obtained from
Table 3 Quantitative measurement by the proposed system

\begin{tabular}{ll}
\hline Parameter & Measured value \\
\hline Power factor & 0.590 \\
Active power & $126.25 \mathrm{~W}$ \\
Apparent power & $213.90 \mathrm{VA}$ \\
Reactive power & $173.23 \mathrm{Var}$ \\
\hline
\end{tabular}

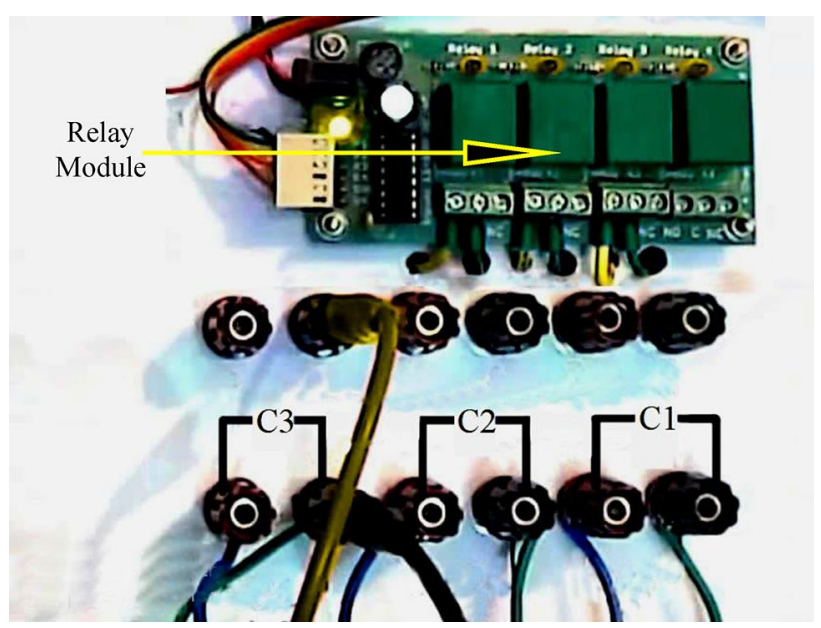

Fig. 18 Experimental set up for power factor improvement

(4). After collecting the instrumental data and finishing some calculations, the system obtains some parameters, as shown in Table 2.
Fig. 17 Power triangle curve generated using measured data from MATLAB

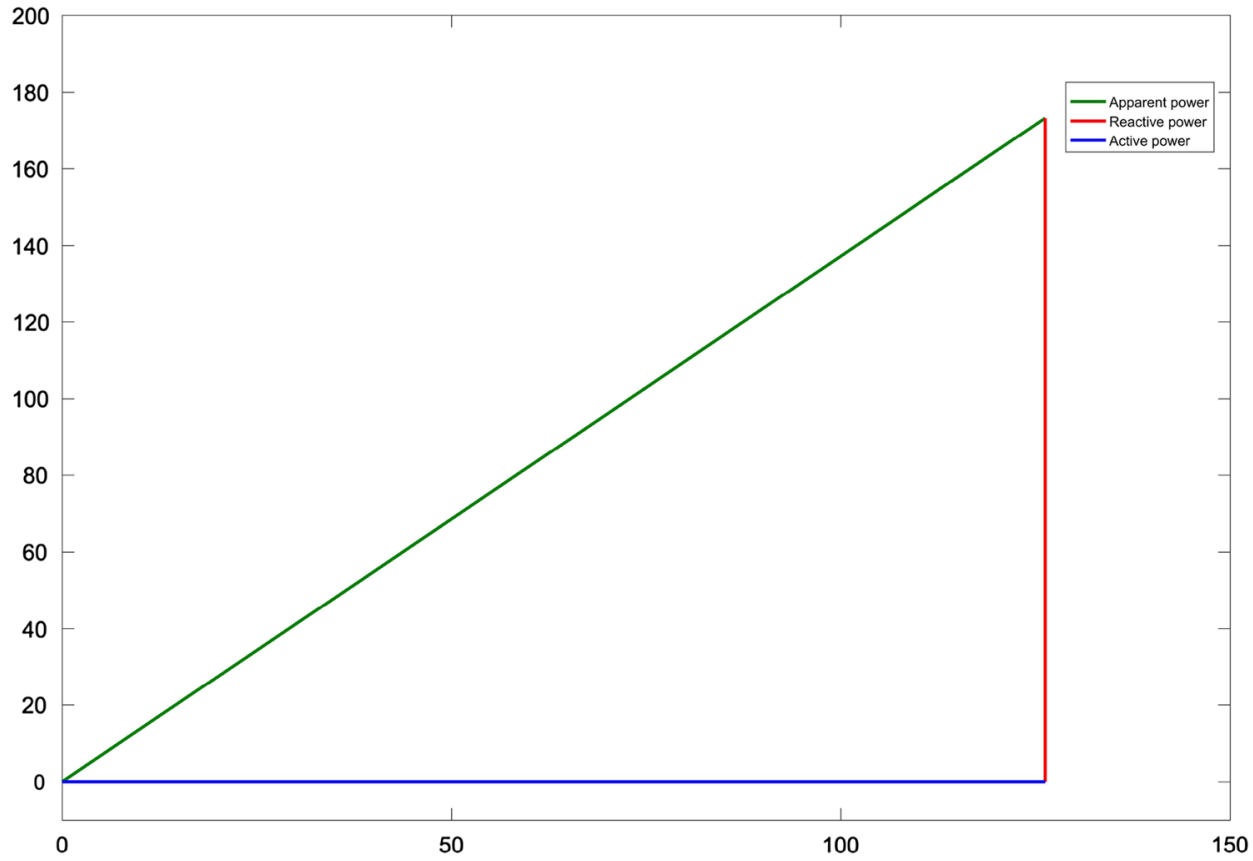

SN Applied Sciences 


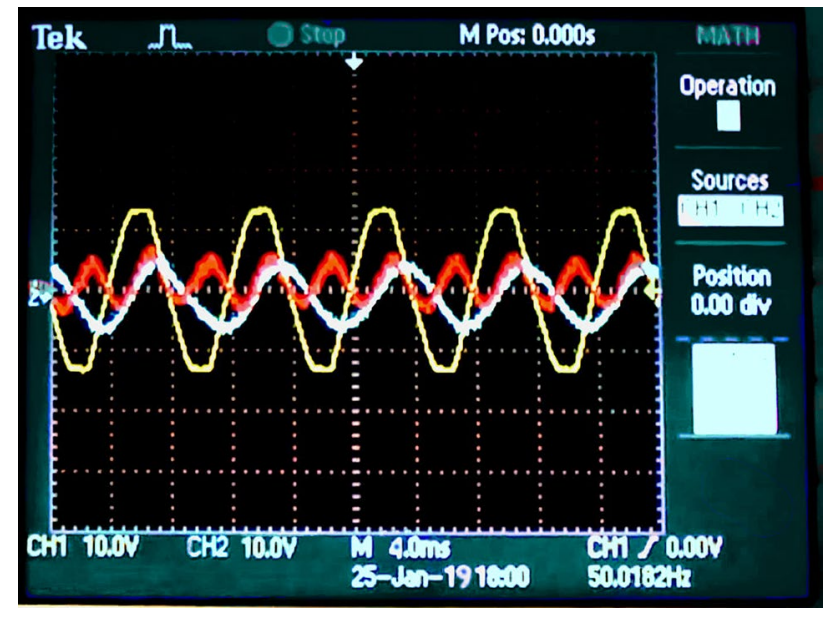

Fig. 19 Experimental improvement results using the proposed system

\subsection{AC power measured by the system}

The obtained results displays on the computer screen where the actual $A C$ power for power factor is calculated by using the proposed system. The MATLAB in proposed system plots a power triangle which is shown in Fig. 17. Here, The green, blue and the red line presents the active, apparent and reactive power respectively. The other calculated parameter is also displayed on the MATLAB command window. Table 3 presents the obtained parameters measured by the proposed system.

After comparing the results, it is observed that the proposed system is able to ensure the required power needed to the load. The proposed system also meets the accurate utility charge within the reasonable range by calculating the appropriate power factor.

\subsection{Power factor corrected by the system}

Figure 18 displays the experimental setup of the power factor improvement system. Three capacitors C1, C2 and $\mathrm{C} 3$ are connected in parallel on the load terminal through the relays. The function of relay is to switch capacitor value during the operation of load. It also ensures the minimum required value for the running of load.

In Fig. 19, the waveforms of the voltage, current and the power is presented after the power factor improvement. The yellow wave shape of the channel represents the voltage, where the white wave shape denotes the current. It is observed that the current is lagging the voltage which originates the lagging power factor. By performing the math operation, the instantaneous power wave shape can be visualize on the oscilloscope. The red wave shape denotes the instantaneous power. The negative portion of

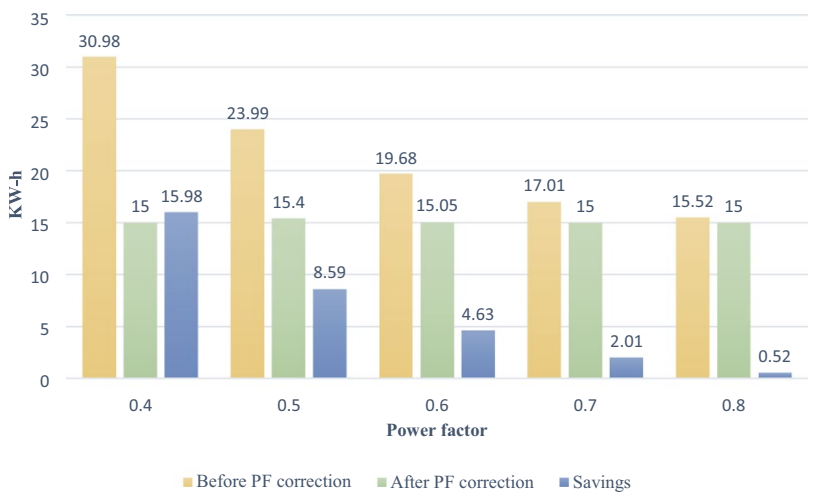

Fig. 20 Comparison of KW-h consumed at different power factor

instantaneous power may be omitted because the current lagging the voltage where the power is always considered as positive value.

\section{Cost analysis}

In this section, the savings from the developed system for an inductive load is analyzed. The optimal design of the power factor improvement system saves money and enhances the utilization of electrical energy. Power factor improvement system recompenses the consumed reactive power and consequently reduces the transmission losses. Most of the cases the consumers need to pay an additional surcharge for the non-usable reactive energy or pays the bill for the apparent electric power.

In this analysis, the size of the power factor improvement unit is selected by considering a $250 \mathrm{~W}$ domestic inductive load. This load is assumed to run at 220 volts. The system first calculates the reactive power required to improve the power factor to 0.9 . At this stage, the end users are not additionally charged with oscillating reactive power. Following this, the required capacitance is calculated and the minimal available capacitance is connected in parallel to the load. The added capacitance supplies the capacitive power which compensates the low power factor.

A comparison of $\mathrm{KW}$-h consumption, between the corrected and uncorrected power factor with the KW-h savings is illustrated in Fig. 20. From the result, it is seen that the $\mathrm{KW}$ - $\mathrm{h}$ savings is more if the load operates with a very low power factor. The charge of the grid and the end user infrastructure is gradually reduced with the increment of the power factor. By assuming the 8 BDT of per unit charge of apparent power, the reactive power to improve the power factor, the required capacitance, the added capacitance to proposed, and the monetary savings are tabulated in Table 4. 
Table 4 Monetary savings at different power factor

\begin{tabular}{|c|c|c|c|c|c|}
\hline Power factor & 0.4 & 0.5 & 0.6 & 0.7 & 0.8 \\
\hline Reactive power (Var) & 451.74 & 311.93 & 212.25 & 133.97 & 66.42 \\
\hline Required Capacitance (uF) & 35.95 & 24.82 & 16.89 & 10.66 & 5.28 \\
\hline Applied capacitance (uF) & 35 & 20 & 15 & 10 & 5 \\
\hline Savings (BDT) & 127.84 & 68.72 & 37.04 & 16.08 & 4.16 \\
\hline
\end{tabular}

Fig. 21 Break-even analysis of the system

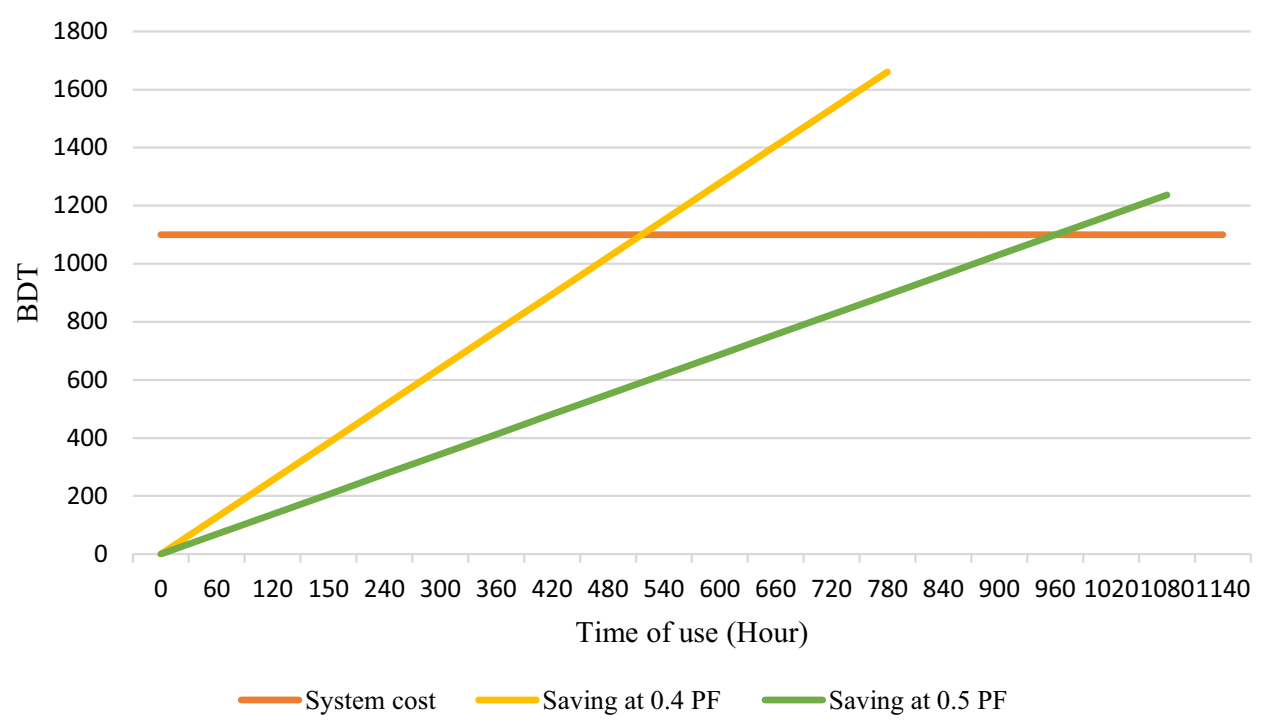

A break-even analysis is shown to obtain the time needed to start making a profit from the proposed power factor correction metering system. The biggest challenge of the system development cost is to estimate which is 1100 BDT approximately. The break-even analysis is shown graphically in Fig. 21. WIth this fixed installation cost, the system needs to provide $540 \mathrm{~h}$ of service with $0.4 \mathrm{PF}$ to make a profit. The profit-making takes a longer time for 0.5 PF. From the break-even analysis, it is manifested that the economic outcome of the system is achieved shortly for a comparatively lower power factor.

A comparison table between the earlier reported approaches and proposed strategy is added based on frequency measurement, PF correction, cost analysis, power triangular visualization, methods implementation complexity in Table 5. References [8-14] has been provided the methodology to correct the PF. These papers have not investigated the other parameters. Frequency measurement, PF correction, cost analysis, power triangular visualization are the main contribution of our paper with respect to the others. It also reduces the utility charge and complexity as compared to the existing system as shown in Table 5.

\section{Conclusion}

A design of high differential strobe terminal based smart power factor correction metering system is presented in this paper for measuring the power factor of an inductive load. This is done by measuring the load voltage, current and time delay between the voltage and current. In many cases, the variation of line frequency limits the calculation of accurate power factor that may result in higher utility charges. The proposed metering system is able to measure the accurate line frequency and find out the appropriate power factor with higher saving of utility charge. The experimental results are used to proof the validation of proposed system. The obtained result confirms the high effectiveness of proposed system. 


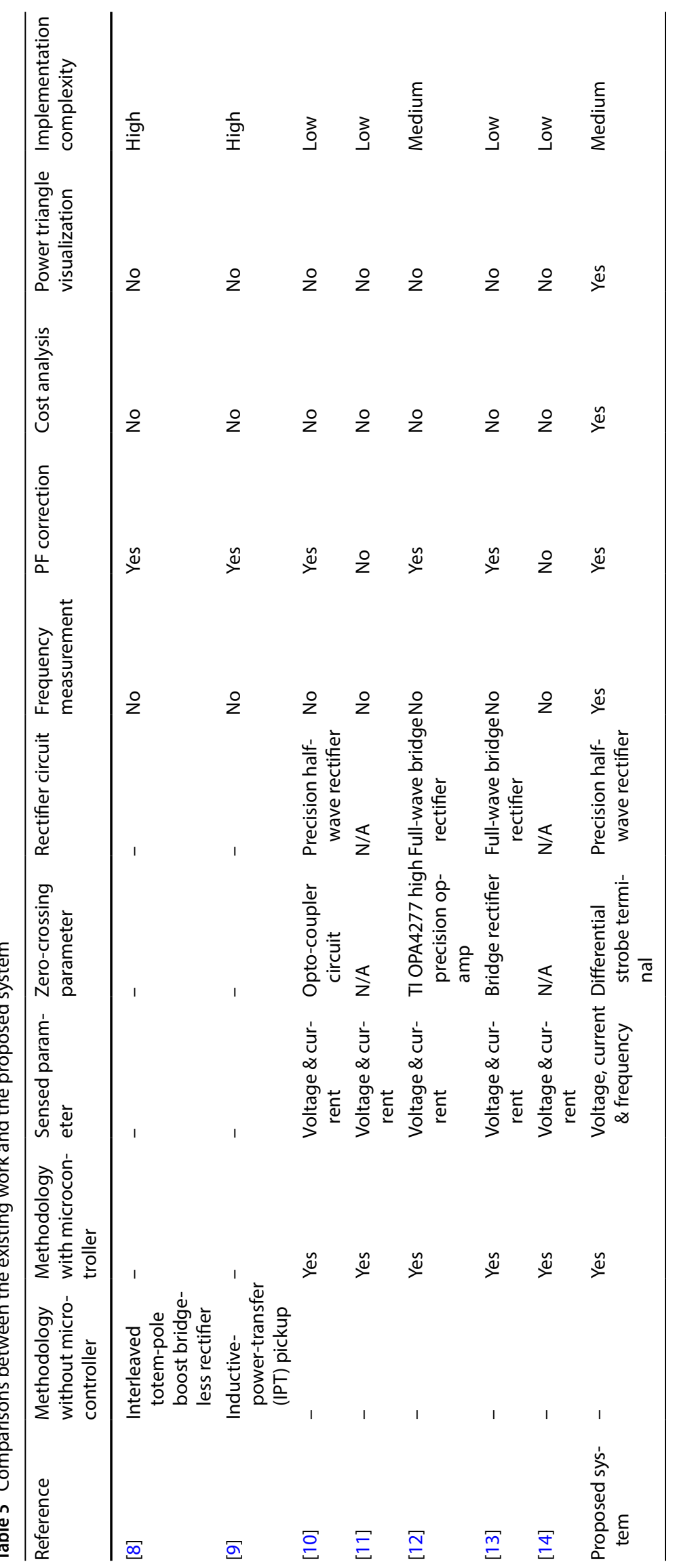


Acknowledgements The authors gratefully thank to Yeahia Sarker and Faisal R. Badal, Department of Mechatronics Engineering, Rajshahi University of Engineering \& Technology, Rajshahi-6204, Bangladesh, for providing their constant support to complete this work.

Funding This study was not funded by any institution or company.

\section{Compliance with ethical standards}

Conflict of interest: The authors declare that they have no conflict of interest.

\section{References}

1. Tamyurek B, Torrey DA (2011) A three-phase unity power factor single-stage ac-dc converter based on an interleaved flyback topology. IEEE Trans Power Electron 26(1):308-318

2. García O, Cobos JA, Prieto R, Alou P, Uceda J (2003) Single phase power factor correction: a survey. IEEE Trans Power Electron 18(3):749-755

3. Grady WM, Santoso S (2001) Understanding power system hannonics. IEEE Power Eng Rev 21(11):8-11

4. Sarker SK, Badal FR, Das P, Das SK (2019) Multivariable integral linear quadratic gaussian robust control of islanded microgrid to mitigate voltage oscillation for improving transient response. Asian J Control 21(4):2114-2125

5. Darly S, Ranjan PV, Bindu K, Rabi BJ (2011) A novel dual boost rectifier for power factor improvement. In: 2011 1st International conference on electrical energy systems. IEEE, pp 122-127

6. Siegler JJ (2014) Systems and methods of increasing power measurement accuracy for power factor correction, May 13, US Patent 8,723,498
7. Wenquan Z, Shaohui C, Jingli Q, Jiansi C (2011) Design of high precision frequency meter based on SOPC. In: 2011 1st International conference on instrumentation, measurement, computer, communication and control. IEEE, pp 566-569

8. Su B, Lu Z (2010) An interleaved totem-pole boost bridgeless rectifier with reduced reverse-recovery problems for power factor correction. IEEE Trans Power Electron 25(6):1406-1415

9. Keeling NA, Covic GA, Boys JT (2010) A unity-power-factor ipt pickup for high-power applications. IEEE Trans Ind Electron 57(2):744-751

10. Khan MB, Owais M (2016) Automatic power factor correction unit. In: 2016 International conference on computing, electronic and electrical engineering (ICE Cube). IEEE, pp 283-288

11. Machado P, Abud T, Fortes M, Borba B (2017) Power factor metering system using Arduino. In: 2017 IEEE workshop on power electronics and power quality applications (PEPQA). IEEE, pp 1-6

12. Saha S, Tyagi T, Gadre DV (2013) Arm (r) microcontroller based automatic power factor monitoring and control system. In: India educators' conference (TIIEC), Texas instruments, 2013. IEEE pp $165-170$

13. Shahid A, Shabir A (2013) Microchip based embedded system design for achievement of high power factor in electrical power systems. In: 2013 IEEE PES Asia-Pacific power and energy engineering conference (APPEEC). IEEE, pp 1-5

14. Dhameliya R, Domadiya K, Miyani P, Savaliya H, Jariwala P (2017) Automatic power factor control using Arduino UNO. Int J Adv Eng Res Dev (IJAERD) 4(4):2348-4470

15. Voltage Comparator $L$ (2012) National semiconductors, texas instruments, literature number: Snosbj1c

Publisher's Note Springer Nature remains neutral with regard to jurisdictional claims in published maps and institutional affiliations. 